\title{
Nontuberculosis mycobacteria infections: would there be pharmacodynamics without pharmacokinetics?
}

\author{
To the Editor:
}

We read with great interest the recent publication by KwAK et al. [1] involving a meta-analysis of studies reporting treatment outcomes for Mycobacterium abscessus pulmonary disease (MAB-PD). The authors should be commended for identifying the association between azithromycin, amikacin and imipenem, and improved clinical outcome in MAB-PD.

M. abscessus, together with M. avium complex (MAC) and M. kansasii, is one of the most common and difficult to treat nontuberculous mycobacteria (NTM) species associated with pulmonary disease [2]. Treatment regimen for NTM infections is largely empirical and driven by clinical experience. Without sound pharmacokinetic (PK)- and pharmacodynamic (PD)-based guidelines on dose, frequency, dose adjustment or monitoring, clinicians are left with uncertainties when it comes to the specifics of prescribing a drug regimen. There is a great need for evidence-based dosing and monitoring strategies.

We would like to kindly alert the authors, other clinicians and researchers about the importance of pharmacokinetics and pharmacodynamics when considering therapeutic effects of drugs. Unfortunately, the authors did not consider collecting PK data, drug exposure (e.g. peak concentration $\left(\mathrm{C}_{\max }\right)$ or area under the concentration time curve AUC)) or therapeutic drug monitoring (TDM) interventions from the original studies. Although CONNELL and WILKIE [3] emphasised the importance of future high-quality studies in their editorial, PK/PD was not addressed. Interestingly, when we looked through the eight original studies included in their final analysis, two studies included PK data. One of them was the study by NAMKOONG et al. [4], in which amikacin dose and weight-adjusted dose $\left(\mathrm{mg} \cdot \mathrm{kg}^{-1}\right)$ was recorded for each patient, and TDM interventions were made based on target trough concentration of $<1 \mu \mathrm{g} \cdot \mathrm{mL}^{-1}$. The other study was by ELLENDER et al. [5], which documented that the median amikacin trough concentration was higher in patients with ototoxicity compared to those without $\left(1.74\right.$ versus $\left.0.92 \mu \mathrm{g} \cdot \mathrm{mL}^{-1} ; \mathrm{p}=0.4\right)$. Although this difference was not statistically significant, possibly due to a small number of patients, potential PK-PD implications cannot be ignored.

The patient's response to an antibiotic is determined by drug exposure at the site of action (affected by absorption, distribution, metabolism and elimination), nature of exposure required (concentration- or time-dependent), and bacteria-related factors such as cellular uptake and presence of resistance genes. Such variable factors may explain why in vitro drug susceptibility is not always correlated with clinical effect in NTM infections [2]. Therefore, minimum inhibitory concentration (MIC) alone should not be used to predict patient's response to therapy, but rather should be interpreted in relation to patient's exposure to the drug, based on $\mathrm{PK} / \mathrm{PD}$ markers such as $\mathrm{AUC} / \mathrm{MIC}, \mathrm{C}_{\max } / \mathrm{MIC}$ or the $\%$ time that drug concentration stays above MIC.

Such PK/PD markers can better account for variability in MIC or drug exposure. MIC ranges for NTM are often higher than those for $M$. tuberculosis, contributing to a relatively low PK/PD target attainment in these patients. KWAK et al. [1] report overall treatment success rate of only 45.6\% for MAB-PD, lower than the rate of culture-conversion. This can potentially be explained by findings from PK/PD studies $[6,7]$.

@ERSpublications

A combination of pharmacokinetics and pharmacodynamics is required to evaluate the efficacy of antimicrobial drugs in the treatment of Mycobacterium abscessus http://bit.ly/2OGwS3C

Cite this article as: Kim HY, Sintchenko V, Alffenaar J-W. Nontuberculosis mycobacteria infections: would there be pharmacodynamics without pharmacokinetics? Eur Respir J 2019; 54: 1901508 [https://doi. org/10.1183/13993003.01508-2019]. 
For amikacin, $\mathrm{C}_{\max } / \mathrm{MIC}$ ratio of 3.20 was associated with $80 \%$ maximum kill rate (EC80) in a hollowfibre model of $M$. abscessus; however, this target was achieved in only $\leqslant 21 \%$ of the simulated patients $(\mathrm{n}=10000)$ with the standard amikacin doses of 750 to $1500 \mathrm{mg}$ per day [7]. Higher $\mathrm{C}_{\max } / \mathrm{MIC}$ and duration of treatment were factors associated with in vitro resistance, highlighting the need for revised regimen based on more clinical data [7]. Inadequate drug exposure in patients with altered PK, such as in cystic fibrosis or due to drug-drug interactions (e.g. clarithromycin, azithromycin), will also result in failure to achieve the required PK/PD target ratio [8].

A combination of macrolide (clarithromycin, azithromycin), amikacin and imipenem is considered standard of care for MAP-PD. New drugs like tigecycline show promising results in PK/PD studies [9]. Unfortunately, the study by KWAK et al. [1] did not allow clinical assessment of this drug due to the limited number that received this agent. As shown in the studies by Ferro et al. [6, 7, 9], optimisation of dosing of current drugs and dose selection for new drugs by PK/PD remains imperative.

Certainly, evidence for TDM has grown stronger in the treatment of M. tuberculosis [10]. Researchers are seeking to find dose-exposure-response relationships and a rationale for TDM in the treatment of NTM infections. For these reasons, as well as due to the challenges of conducting prospective TDM studies, we believe that paired PK-PD data from studies integrated by KwAK et al. [1] could have yielded valuable information, as exemplified by VAN INGEN et al. [8] in their PK-PD analysis of patients with MAC infection. Further research in this area to facilitate establishment of PK/PD target, specific reference ranges and patient tailored dosing to optimise treatment of NTM infections is warranted.

Hannah Yejin Kim ${ }^{1,2}$, Vitali Sintchenko ${ }^{3,4,5}$ and Jan-Willem Alffenaar ${ }^{1,2}$

${ }^{1}$ Sydney Pharmacy School, Faculty of Medicine and Health, The University of Sydney, Sydney, Australia. ${ }^{2}$ Westmead Hospital, Sydney, Australia. ${ }^{3}$ Sydney Medical School, The University of Sydney, Sydney, Australia. ${ }^{4}$ Centenary Institute, The University of Sydney, Sydney, Australia. ${ }^{5}$ Centre for Infectious Diseases and Microbiology-Public Health, Westmead Hospital, Sydney, Australia.

Correspondence: Jan-Willem Alffenaar, Sydney Pharmacy School, Faculty of Medicine and Health, The University of Sydney, Sydney, NSW, 2006, Australia. E-mail: johannes.alffenaar@sydney.edu.au

Received: 30 July 2019 | Accepted: 01 Aug 2019

Conflict of interest: None declared.

\section{References}

1 Kwak N, Dalcolmo MP, Daley CL, et al. Mycobacterium abscessus pulmonary disease: individual patient data meta-analysis. Eur Respir J 2019; 54: 1801991.

2 Cowman S, van Ingen J, Griffith DE, et al. Non-tuberculous mycobacterial pulmonary disease. Eur Respir J 2019; 54: 1900250.

3 Connell DW, Wilkie M. Pulmonary Mycobacterium abscessus: can we identify the road to improved outcomes? Eur Respir J 2019; 54: 1901121.

4 Namkoong H, Morimoto K, Nishimura T, et al. Clinical efficacy and safety of multidrug therapy including thrice weekly intravenous amikacin administration for Mycobacterium abscessus pulmonary disease in outpatient settings: a case series. BMC Infect Dis 2016; 16: 396.

5 Ellender CM, Law DB, Thomson RM, et al. Safety of IV amikacin in the treatment of pulmonary non-tuberculous mycobacterial disease. Respirology 2016; 21: 357-362.

6 Ferro BE, Srivastava S, Deshpande D, et al. Failure of the amikacin, cefoxitin, and clarithromycin combination regimen for treating pulmonary Mycobacterium abscessus infection. Antimicrob Agents Chemother 2016; 60: 6374-6376.

7 Ferro BE, Srivastava S, Deshpande D, et al. Amikacin pharmacokinetics/pharmacodynamics in a novel hollow-fiber Mycobacterium abscessus disease model. Antimicrob Agents Chemother 2015; 60: 1242-1248.

8 van Ingen $\mathrm{J}$, Egelund $\mathrm{EF}$, Levin $\mathrm{A}$, et al. The pharmacokinetics and pharmacodynamics of pulmonary Mycobacterium avium complex disease treatment. Am J Respir Crit Care Med 2012; 186: 559-565.

9 Ferro BE, Srivastava S, Deshpande D, et al. Tigecycline is highly efficacious against Mycobacterium abscessus pulmonary disease. Antimicrob Agents Chemother 2016; 60: 2895-2900.

10 Ghimire S, Bolhuis MS, Sturkenboom MG, et al. Incorporating therapeutic drug monitoring into the World Health Organization hierarchy of tuberculosis diagnostics. Eur Respir J 2016; 47: 1867-1869.

Copyright @ERS 2019

From the authors:

We appreciate the comments from H.Y. Kim and co-workers on our individual patient data meta-analysis on Mycobacterium abscessus pulmonary disease (MAB-PD) [1]. They emphasised the importance of pharmacokinetic (PK)/pharmacodynamic (PD) analysis and therapeutic drug monitoring (TDM) in the 
field of nontuberculous mycobacterial (NTM) disease, especially for MAB-PD. We generally agree with their perspective.

As H.Y. Kim and co-workers pointed out, most of the recommendations for MAB-PD treatment stem from empirical data and experts' opinions. In our study [1], we reported the importance of azithromycin, amikacin and imipenem in the treatment of M. abscessus subspecies abscessus. However, optimum doses of these drugs have not yet been firmly established. While the American Thoracic Society/Infectious Diseases Society of America recommended the daily use of $250 \mathrm{mg}$ azithromycin [2], the British Thoracic Society suggested a dosage of up to $500 \mathrm{mg}$ daily [3]. The use of amikacin has been recommended with variable dosing, from $7-10 \mathrm{mg} \cdot \mathrm{kg}^{-1}$ to $10-30 \mathrm{mg} \cdot \mathrm{kg}^{-1}$, depending on the frequency of administration, and the targeted peak concentrations $\left(C_{\max }\right)$ have also varied from $20-25 \mu \mathrm{g} \cdot \mathrm{mL}^{-1}$ to $35-45 \mu \mathrm{g} \cdot \mathrm{mL}^{-1}$ [4]. These variable suggestions underscore the importance of PK/PD analysis in the treatment of NTM pulmonary disease. Although two out of eight studies included in our analysis reported PK data, as H.Y. Kim and co-workers indicated, we were unable to include these PK data in our meta-analysis because other studies lacked such information.

Previously, vAN INGEN et al. [5] reported the PK/PD parameters in patients with Mycobacterium avium complex pulmonary disease (MAC-PD) ( . van Ingen is one of the co-authors of our study). $\mathrm{C}_{\max }$ and areas under the time-concentration curve (AUC) were measured in 531 patients and the minimum inhibitory concentration (MIC) of their isolates analysed. Although these patients were being treated with currently recommended doses, the desirable target ratio of $\mathrm{C}_{\max } / \mathrm{MIC}$ or AUC/MIC for clarithromycin and amikacin were attained only in $42 \%$ and $19 \%$ of patients, respectively.

In the case of $M$. abscessus, incubation at sub-inhibitory concentrations of macrolides resulted in a marked increase of MIC related to inducible resistance [6]. Prolonged incubation of M. abscessus with exposure to $1 \times$ MIC of either clarithromycin or azithromycin yielded a $>30$-fold increase in the MIC as well as increased expression of erm(41) after 7 days of inoculation. In addition, the sub-inhibitory doses of amikacin changed the phenotypes of $M$. abscessus from smooth to rough colony morphology [7]. Defining optimal dosage of core drugs based on PK/PD analysis is pivotal to improving treatment outcomes of MAB-PD. However, the current regimens were suggested by results of in vitro studies and the data from several case series $[2,3]$. PK/PD analysis in real-life clinical practice is somewhat tricky because of the complexity of treatment, including use of multiple drugs and toxicity issues limiting the choice and dosing of key antibiotics [2,3]. The hollow-fibre system models using Monte Carlo simulations could be an alternative to conventional PK/PD study in humans [8].

LEE et al. [4] reported their experience using TDM for patients with MAB-PD (W-J. Koh, a co-author with LEE et al. [4], is another of the co-authors of our study). They adjusted the amikacin dose to achieve the target $\mathrm{C}_{\max }$ of $55-65 \mu \mathrm{g} \cdot \mathrm{mL}^{-1}$. In week 4,46 out of 48 patients $(95.8 \%)$ achieved the target level, while transient nephrotoxicity developed in $6.3 \%$ of patients and ototoxicity in $25.0 \%$. Although the treatment of MAB-PD necessitates the long-term use of intravenous drugs, safety and tolerability can be achieved if TDM is applied.

One pitfall in PK/PD analysis and TDM is the uncertain correlation with treatment outcomes. These stem from various reasons, including use of multiple drugs, the time- rather than concentration-dependent activity of some key antimycobacterial drugs (cefoxitin, imipenem and macrolides) [8] and the fact that rifampicin and ethambutol feature in MAC-PD regimens mainly for their synergy and to prevent macrolide resistance, not for their killing capacity [2, 3]. In MAC-PD, low plasma clarithromycin concentration was reported not to be associated with treatment outcomes [9], although subsequent study reported that a higher $C_{\max }$ of azithromycin was associated with favourable outcomes [10]. The proportion of patients satisfying the PD target for amikacin was 19\%, which could not explain the treatment success rates for MAC-PD reaching 60\% [5]. These data suggested that the clinical implication of PK/PD parameters must be interpreted with caution. Future studies such as the multinational FORMAT trial (Finding the Optimum Regimen for Mycobacterium abscessus) will include PK/PD analysis of all drugs, combined with clinical and microbiological correlations, to improve treatment outcomes of MAB-PD.

@ERSpublications

Pharmacokinetic, pharmacodynamic and therapeutic drug monitoring data are useful in optimising treatment for Mycobacteriuam abscessus pulmonary disease, but such data are not routinely collected and analysis for clinical practice is difficult http://bit.ly/2pcTIUn

Cite this article as: Kwak N, Dalcolmo MP, Daley CL, et al. Nontuberculosis mycobacteria infections: would there be pharmacodynamics without pharmacokinetics? Eur Respir J 2019; 54: 1901806 [https://doi. org/10.1183/13993003.01806-2019]. 
Nakwon Kwak ${ }^{1}$, Margareth Pretti Dalcolmo ${ }^{2}$, Charles L. Daley ${ }^{3}$, Geoffrey Eather ${ }^{4}$, Naoki Hasegawa ${ }^{5}$, Won-Jung Koh $\oplus^{6}$, Rachel Thomson ${ }^{7}$, Jakko van Ingen $\oplus^{8}$ and Jae-Joon Yim ${ }^{1}$

${ }^{1}$ Division of Pulmonary and Critical Care Medicine, Dept of Internal Medicine, Seoul National University College of Medicine, Seoul, South Korea. ${ }^{2}$ Centro de Referência Professor Hélio Fraga, Escola Nacional de Saúde Pública Sérgio Arouca, Fundação Oswaldo Cruz, Rio de Janeiro, Brazil. ${ }^{3}$ Division of Mycobacterial and Respiratory Infections, Dept of Medicine, National Jewish Health, Denver, CO, USA. ${ }^{4}$ Metro South Clinical Tuberculosis Service and Dept of Respiratory and Sleep Medicine, Princess Alexandra Hospital, Brisbane, Australia. ${ }^{5}$ Keio University School of Medicine, Center for Infectious Disease and Infection Control, Tokyo, Japan. ${ }^{6}$ Division of Pulmonary and Critical Care Medicine, Dept of Medicine, Samsung Medical Center, Sungkyunkwan University School of Medicine, Seoul, South Korea. ${ }^{7}$ Gallipoli Medical Research Institute, University of Queensland, Brisbane, Australia. ${ }^{8}$ Dept of Medical Microbiology, Radboud University Medical Center, Nijmegen, The Netherlands.

Correspondence: Jae-Joon Yim, Division of Pulmonary and Critical Care Medicine, Dept of Internal Medicine, Seoul National University College of Medicine, 101 Daehak-Ro, Jongno-Gu, Seoul 110-744, South Korea. E-mail: yimjj@snu.ac.kr

Received: 13 Sept 2019 | Accepted: 13 Sept 2019

Author contributions: The authors meet criteria for authorship as recommended by the International Committee of Medical Journal Editors, were fully responsible for all content, and were involved at all stages of manuscript development.

Conflict of interest: N. Kwak has nothing to disclose. M.P. Dalcolmo has nothing to disclose. C.L. Daley reports grants from Insmed, and personal fees from Insmed, Johnson \& Johnson, Spero, Paratek and Horizon, outside the submitted work. G. Eather has nothing to disclose. N. Hasegawa reports a grant for a clinical trial and personal fees for consultation from Insmed, outside the submitted work. W-J. Koh reports personal fees for advisory boards from Johnson \& Johnson and Insmed, outside the submitted work. R. Thomson reports personal fees from Insmed and Savara for advisory boards, and from Menarini for CME talks, outside the submitted work. J. van Ingen reports funding from Insmed, Janssen Pharmaceuticals, Spero Therapeutics and Paratek for advisory boards, during the conduct of the study. J-J. Yim has nothing to disclose.

\section{References}

1 Kwak N, Dalcolmo MP, Daley CL, et al. Mycobacterium abscessus pulmonary disease: individual patient data meta-analysis. Eur Respir J 2019; 54: 1801991.

2 Griffith DE, Aksamit T, Brown-Elliott BA, et al. An official ATS/IDSA statement: diagnosis, treatment, and prevention of nontuberculous mycobacterial diseases. Am J Respir Crit Care Med 2007; 175: 367-416.

3 Haworth CS, Banks J, Capstick T, et al. British Thoracic Society guidelines for the management of non-tuberculous mycobacterial pulmonary disease (NTM-PD). Thorax 2017; 72: Suppl. 2, ii1-ii64.

4 Lee H, Sohn YM, Ko JY, et al. Once-daily dosing of amikacin for treatment of Mycobacterium abscessus lung disease. Int J Tuberc Lung Dis 2017; 21: 818-824.

5 van Ingen $\mathrm{J}$, Egelund $\mathrm{EF}$, Levin $\mathrm{A}$, et al. The pharmacokinetics and pharmacodynamics of pulmonary Mycobacterium avium complex disease treatment. Am J Respir Crit Care Med 2012; 186: 559-565.

6 Schildkraut JA, Pennings LJ, Ruth MM, et al. The differential effect of clarithromycin and azithromycin on induction of macrolide resistance in Mycobacterium abscessus. Future Microbiol 2019; 14: 749-755.

7 Tsai SH, Lai HC, Hu ST. Subinhibitory doses of aminoglycoside antibiotics induce changes in the phenotype of Mycobacterium abscessus. Antimicrob Agents Chemother 2015; 59: 6161-6169.

8 Ferro BE, Srivastava S, Deshpande D, et al. Failure of the amikacin, cefoxitin, and clarithromycin combination regimen for treating pulmonary Mycobacterium abscessus infection. Antimicrob Agents Chemother 2016; 60: 6374-6376.

9 Koh WJ, Jeong BH, Jeon $\mathrm{K}$, et al. Therapeutic drug monitoring in the treatment of Mycobacterium avium complex lung disease. Am J Respir Crit Care Med 2012; 186: 797-802.

10 Jeong $\mathrm{BH}$, Jeon $\mathrm{K}$, Park HY, et al. Peak plasma concentration of azithromycin and treatment responses in Mycobacterium avium complex lung disease. Antimicrob Agents Chemother 2016; 60: 6076-6083. 